\title{
SOME RESULTS IN DOEBLIN'S THEORY OF MARKOV CHAINS ${ }^{1}$
}

\author{
BY WILLIAM WINKLER
}

Communicated by Jacob Feldman, October 26, 1973

\begin{abstract}
Our notation and definitions are taken from Chung [1]. A closed set $H$ is called recurrent in the sense of Harris if there exists a $\sigma$-finite measure $\varphi$ such that for $E \subseteq H, \varphi(E)>0$ implies $Q(x, E)=1$ for all $x \in H$.

THEOREM 1. Let $X$ be absolutely essential and indecomposable. Then there exists a closed set $B \subseteq X$ such that $B$ contains no uncountable disjoint collection of perpetuable sets if and only if $\mathrm{X}=\mathrm{H}+\mathrm{I}$ where $H$ is recurrent in the sense of Harris and I is either inessential or improperly essential.

THEOREM 2. If there exists no uncountable disjoint collection of closed sets, then there exists a countable disjoint collection $\left\{D_{n}\right\}_{n=1}^{\infty}$ of absolutely essential and indecomposable closed sets such that $I=X-\sum_{n=1}^{\infty} D_{n}$ is either inessential or improperly essential.
\end{abstract}

Under the additional assumption that Suslin's conjecture holds, Theorem 2 was proved by Jamison [7].

In this announcement we present two theorems which show that a major portion of Doeblin's and Harris' theory may be derived without making the standard assumptions about the reference measure which have characterized this theory (see [8, p. 4], [1], [2], [3], [4], [5], [6]). The second theorem is due to Jamison under the additional assumption that Suslin's conjecture holds. Our notation and definitions are taken from [1]. A closed set $H \subseteq X$ is called recurrent in the sense of Harris if there exists a $\sigma$-finite measure $\varphi$ such that if $E \subseteq H$ where $\varphi(E)>0$, then $Q(x, E)=1$.

THEOREM 1. Let $X$ be absolutely essential and indecomposable. Then the following are equivalent:

(i) There exists a closed set $B$ such that $B$ contains no uncountable disjoint collection of perpetuable sets.

(ii) $X$ is normal.

(iii) $X=H+I$ where $H$ is recurrent in the sense of Harris and I is either inessential or improperly essential.

AMS (MOS) subject classifications (1970). Primary 60JXX.

1 This paper is based on a part of the author's Ph.D. thesis prepared at the Ohio State University under the direction of Professor Louis Sucheston. 
SKETCH OF PROOF. The equivalence of (ii) and (iii) is known (see [5], [6]). (iii) implies (i) follows from [1, Proposition 21], and the fact that there exists a $\sigma$-finite invariant measure $\pi$ on $H$ such that if $E \subseteq H$, then $\pi(E)=0$ if and only if $E$ is inessential. In order to prove that (i) implies (ii) we use [1, Proposition 23.1] and transfinite induction.

THEOREM 2. If there exists no uncountable disjoint collection of closed sets, then there exists a countable collection $\left\{D_{n}\right\}_{n=1}^{\infty}$ of absolutely essential and indecomposable closed sets such that $I=X-\sum_{n=1}^{\infty} D_{n}$ is either inessential or improperly essential.

SKETCH OF PROOF. There exists a function $C(\cdot)$ from the binary sequences into \{closed subsets of $X\} \cup\{\varnothing\}$ such that

(a) $C(s)$ is either closed or empty,

(b) if $s \subseteq t$, then $C(t) \subseteq C(s)$,

(c) if neither $s \subseteq t$ nor $t \subseteq s$, then $C(s) \cap C(t)=\varnothing$ (see [6, p. 289]). Define $\mathscr{R}=\{C(s): s$ binary sequence $\}-\{\varnothing\}$. Let $\Omega$ be the first uncountable ordinal. For each ordinal $\beta<\Omega$, let $K(\beta)=\bigcup\{C(s) \in \mathscr{R}$ : order $s=\beta\}$. Theorem 2 follows from an argument using the construction of a $\sigma$ finite measure $m$ such that $m(K(\beta))>0$ for all $\beta<\Omega$, and the following

Lemma. Assume that every closed set is absolutely essential and decomposable and there exists no uncountable disjoint collection of closed sets. Then there exists no $\sigma$-finite measure $\varphi$ such that $C \in \mathscr{R}$ implies $m(C)>0$.

\section{REFERENCES}

1. K. L. Chung, The general theory of Markov processes according to Doeblin, Z. Wahrscheinlichkeitstheorie und Verw. Gebiete 2 (1964), 230-254. MR 29 \#4108.

2. W. Doeblin, Éléments d'une théorie générale des chaînes simples constantes de Markoff, Ann. Sci. Ecole Norm. Sup. (3) 57 (1940), 61-111. MR 3, 3.

3. T. E. Harris, The existence of stationary measures for certain Markov processes, Proc. Third Berkeley Sympos. Math. Statist. and Probability (1954/55), vol. II, Univ. of California Press, Berkeley, Calif., 1956, pp. 113-124. MR 18, 941.

4. - Correction to a proof, $\mathrm{Z}$. Wahrscheinlichkeitstheorie und Verw. Gebiete 12 (1969), 172. MR 39 \#7665.

5. N. C. Jain, Some limit theorems for a general Markov process, Z. Wahrscheinlichkeitstheorie und Verw. Gebiete 6 (1966), 206-223. MR 35 \#7411.

6. N. Jain and B. Jamison, Contributions to Doeblin's theory of Markov processes, Z. Wahrscheinlichkeitstheorie und Verw. Gebiete 8 (1967), 19-40. MR 36 \#4643.

7. B. Jamison, A result in Doeblin's theory of Markov chains implied by Suslin's conjecture, Z. Wahrscheinlichkeitstheorie und Verw. Gebiete 24 (1972), 287-293.

8. S. Orey, Limit theorems for Markov chain transition probabilities, Van Nostrand Math. Studies, no. 34, Van Nostrand-Reinhold, London 1971.

Department of Mathematics, Ohio State University, Columbus, Ohio 43210

Current address: Department of Mathematics, State University of New York at Albany, Albany, New York 12222 